\title{
Applying Advanced NN-based Decision Support Scheme for Heart Diseases Diagnosis
}

\author{
Sameh Ghwanmeh \\ Computer Science Department \\ Faculty of IT \\ WISE University \\ Jordan
}

\begin{abstract}
Heart diagnosis equipment is not always available in every medical center, especially in the rural areas where less support and care. Physician intuition and experience are not always enough to attain high quality medical results. Therefore, medical errors and unwanted results are reasons for a need for unconventional computer-based diagnosis systems, which in turns reduce medical fatal errors, increasing the patient safety and save lives. The proposed system, which is based on Artificial Neural Networks (ANNs), provides a decision support system to classify the heart diseases: mitral stenosis, aortic stenosis and ventricular septal defect. Additionally, the system offers a promising opportunity to develop an operational screening and testing device for heart disease diagnosis and can provide great assistance for clinicians to make advanced heart sound diagnosis, especially in rural areas where high tech devices may not be available. Series of experiments have been conducted, using real medical data, to test the performance and accuracy of the proposed system. Compared results revealed that the system performance and accuracy are excellent, with a heart diseases classification accuracy of $92 \%$.
\end{abstract}

\section{General Terms}

Neural Networks, Diagnosis Systems, Heart Diseases

Classification.

\section{Keywords}

ANNs, decision support system, heart disease diagnosis, knowledge base, classification accuracy.

\section{INTRODUCTION}

The human heart is a compound body organ contains muscles together with biological nerves. The heart pumps nearly 51 of blood in the body providing the human body with renewed materials [1]. Medical research literature shows that there is much interest from the scientific researchers in implementing the human intelligence, including ANNs, in medical devices. The human heart operation is composite and any failure is risky to human lives. Therefore, heart diagnose systems has been a main concern to the scientific researchers in the last decades [2]. It is not possible to use linear systems to perform the heart diagnosis. ANN is a non-linear behavior structure and has been proven to be effective in the diagnosis systems [3].

Heart diagnosis equipment is not always available in every medical center, especially in the rural areas where less support and care. Additionally, it is not possible for many people to travel to regional medical centers where high quality hospital services are affordable. Physician intuition and experience are not always enough to attain high quality medical results. Therefore, Medical errors and unwanted results are reasons for a need for innovative computer-based diagnosis systems, which in turns reduce medical fatal errors, increase patient safety and save lives [4,5]. Additionally, intuition and experience are frequently used by physicians to reach the medical verdicts instead of knowledge base. Consequently, unsolicited preconceptions and excessive medical cost are produced which may portend the patients' lives $[6,7]$.

Generally, ANNs have been used in many areas of medicine, such as cardiology, Electroencephalography [8], Pulmonology [9], Genetics, Clinical chemistry, Pathology, Ophthalmology, Obstetrics and Gynecology [10]. Further, considerable research has been conducted on heart diagnosis $[11,12]$. However, most of these studies deal with only one specific disease area, dedicated to valvular diseases. The following provides an overview of related studies:

- Yana et al. presents "A multilayer perceptron-based medical decision support system for heart disease diagnosis" study. The study deals with identifying 5 heart diseases based on heart auscultation: hypertension, coronary heart disease, rheumatic valvular heart disease, chronic cor pulmonale and congenital heart disease [9].

- $\quad$ Tripathy presents "System for Diagnosing Valvular Heart Disease using Heart Sounds" study. The study deals with a diagnosis scheme based on heart auscultation for aortic regurgitation, aortic stenosis, mitral regurgitation and mitral stenosis [13].

- Zamri presents "Wavelet Analysis and Classification of Mitral Regurgitation and Normal Heart Sounds" study. The study deals with mitral regurgitation diagnose based on ANNs [14].

- Turkoglu and Arslan present "An Intelligent Pattern Recognition System Based on Neural Network and Wavelet Decomposition for interpretation of heart sounds" study. The study deals with aortic stenosis, mitral regurgitation, atrial septal defect mitral stenosis and aortic regurgitation disease diagnosis [15].

- Other related studies can be found in $[9,16,17]$.

In this research, it is proposed to build an ANN decision support system for heart diseases diagnosis. The system contains two main components: a software part and a hardware part, which consists of a simple electronic stethoscope with a matching impedance electronic circuit. It is expected that the proposed system would benefit medical physicians to diagnose heart sound signals and checking the up-normality. Also, it is anticipated that the proposed system would provide innovative diagnostic tool to classify the heart 
diseases: mitral stenosis (MS), aortic stenosis (AS) and ventricular septal defect (ASD). Additionally, the system would offer a promising opportunity to develop an operational screening and testing device for heart disease diagnosis.

The performance, classification accuracy and effectiveness of the proposed ANN-based decision support system have been examined. Series of experiments were carried out using real medical data from the Royal Medical Services. The testing experiments have been conducted to provide a clear comparison between the proposed system and other systems and techniques. Performance evaluation process has been performed to reveal the best value of heart diseases classification accuracy.

\section{RESEARCH METHODOLOGY}

The proposed ANN decision support system is composed of two parts: hardware and GUI software. The hardware is constructed to represent the pattern recognition sensor to collect heart sounds. Since the collected sound by stethoscope is low amplitude level, a microphone capsule is inserted between the stethoscope and the PCI sound card interface to amplify the sound waves fed by the stethoscope. The GUI software part is implemented using Matlab and NN toolbox.

\subsection{Data Collection}

The testing data have been collected based on two different sources. The first source is provided by the Jordanian Royal Medical Services. While the second source is the recorded samples directly provided from the patient tested on the proposed system.

\subsection{Extracting the Signal Features}

The Matlab FFT function is used to extract the main features of the sound. The FFT assumptions employed in the proposed system are shown below.

- $\quad$ The sample frequency was set to $8000 \mathrm{~Hz}$.

- Perform FFT on the wave file (100,000 samples are taken).

- Taking the absolute value of the important (beginning 5000) part of data in the new matrix.

- Split the FFT matrix into 50 bins and get the average of each.

- Standardize the return value by dividing the matrix by its sum.

\subsection{Training of Neural Networks}

Three NNs are built: mitral stenosis disease NN, ventricular septal defect disease NN and aortic stenosis disease NN. The NNs parameters are initialized based on default values, including: epochs (number of iterations), goal (performance function) and $\mathrm{NN}$ show number.

\section{RESULTS AND DISCUSSION}

Series of experiments with different samples have been conducted to measure the effectiveness and accurateness of the proposed system. To ensure the correctness of the knowledge base and outputs, $\mathrm{NN}$ are trained repeatedly and its parameters are modified where is needed. Number of trained samples, with wide range of variations and noise effect in each class, are also increased to enhance the system accuracy. Results show that accurate outputs, with $98 \%$ classification accuracy, are resulted with the trained samples, while, the system produces acceptable results for non-trained samples, with $92 \%$ classification accuracy.

Figure 1 shows the results for a normal heart, while Figure 2 presents the up-normal heart "arterial stenosis". Clearly, the analog waves are different based on the trained samples in the NN knowledge base. Figure 3, Figure 4 and Figure 5 depicts the results for patients with MS, AS and VSD heart diseases, respectively. It can be seen that $\mathrm{NN}$ trained samples yield accurate heart disease identification, with $98 \%$ classification accuracy. While, for non-trained samples, the system yield results with $92 \%$ classification accuracy.

Additional experiments are carried out using external stethoscope and the output is accurate. However, it can be seen (Fig.6) that the shape of the recorded sound waveform looks little different compared with the stored one. The reason behind this is that the samples waveforms were sampled at different rate.

Overall, the results revealed that all heart diseases of MS, AS and VSD are successfully and accurately identified with the proposed system, as shown in (Fig.1, Fig.2, Fig.3, Fig.4, Fig.5, Fig.6). Compared with previous studies presented in $[9,13,15,18]$ which are mainly focused on valvular heart diseases, the proposed system delivers higher classification accuracy for both valvular and non-valvular heart diseases.

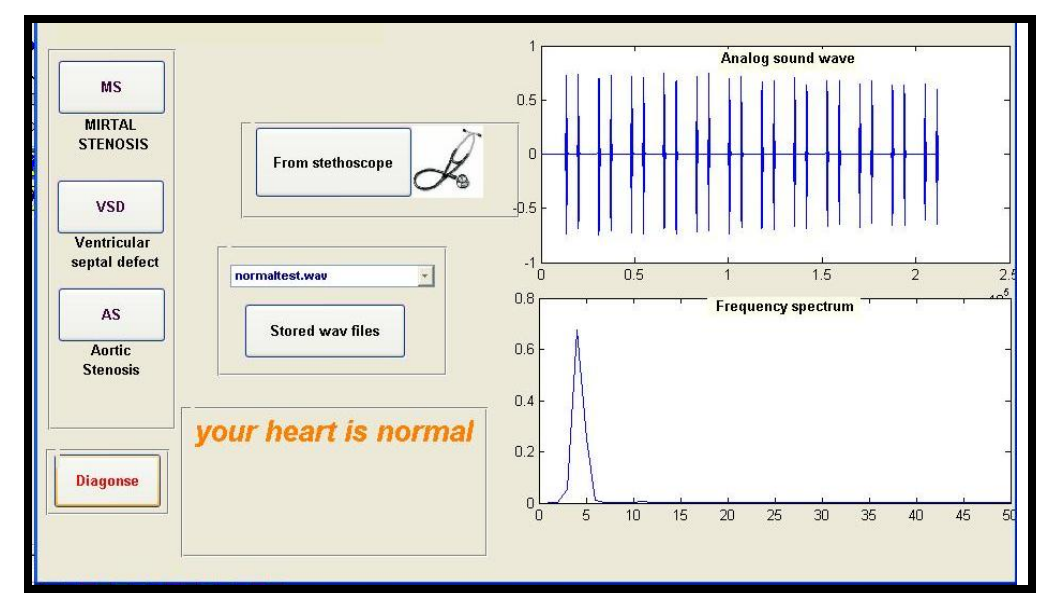

Fig 1: System Output for "normal heart". 


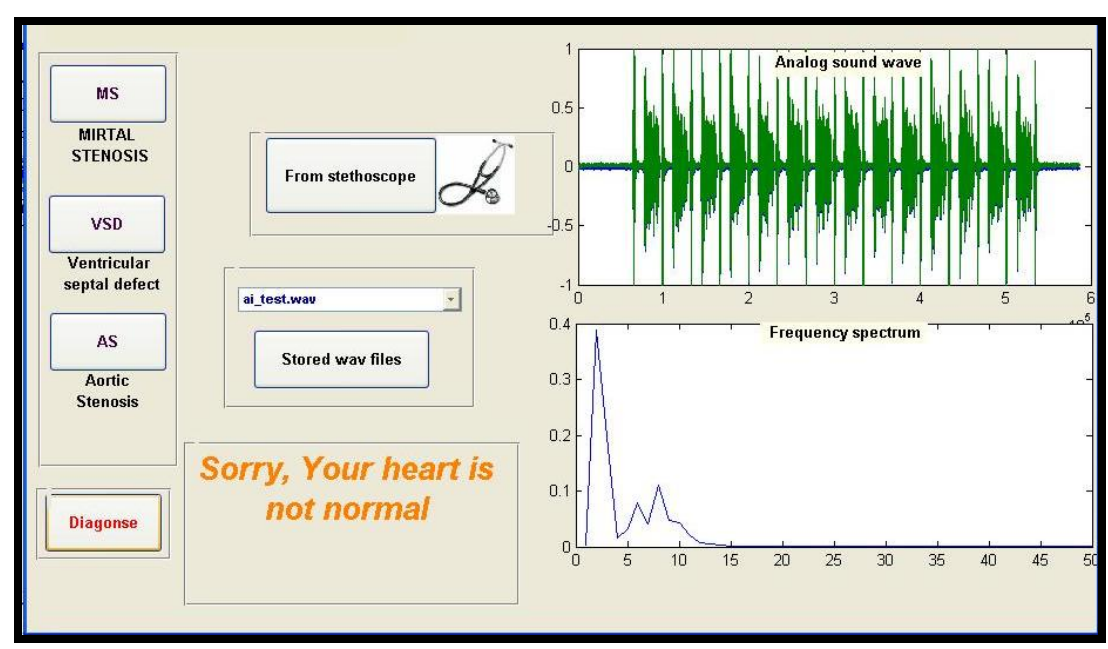

Fig 2: System Output for up-normal heart "arterial stenosis".

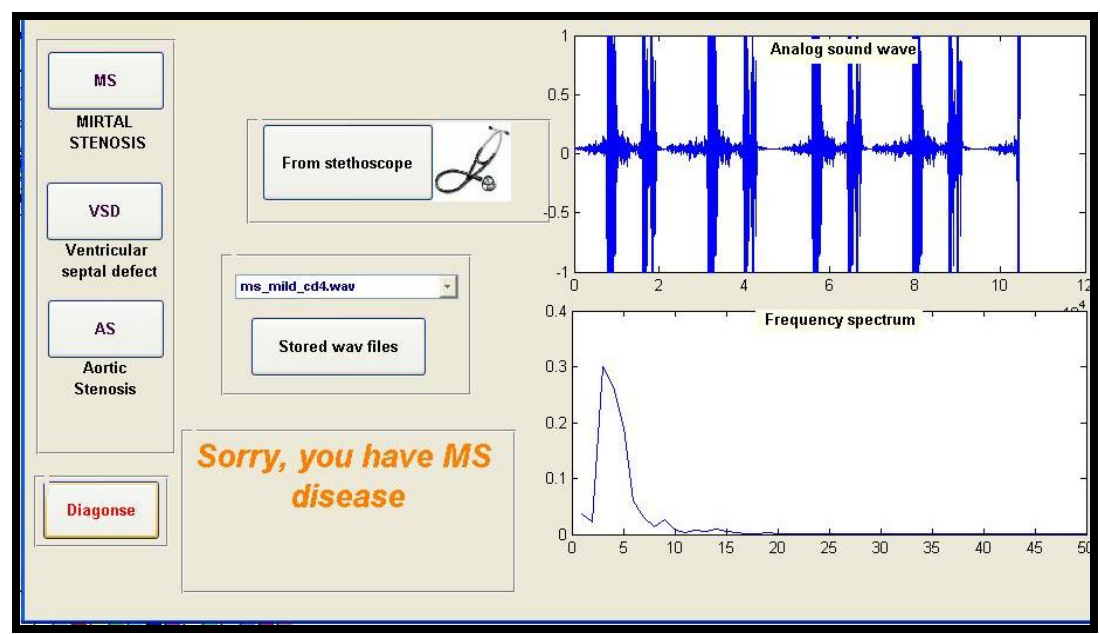

Fig 3: System Output for "mitral stenosis" heart disease.

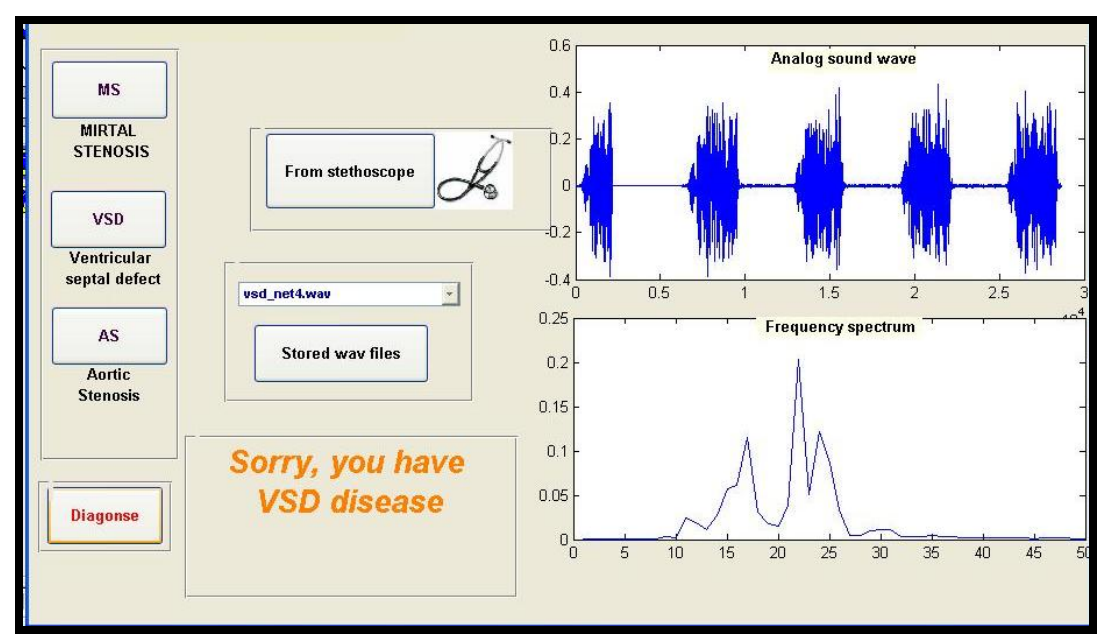

Fig 4: System Output for "Ventricular Septal Defect" heart disease. 


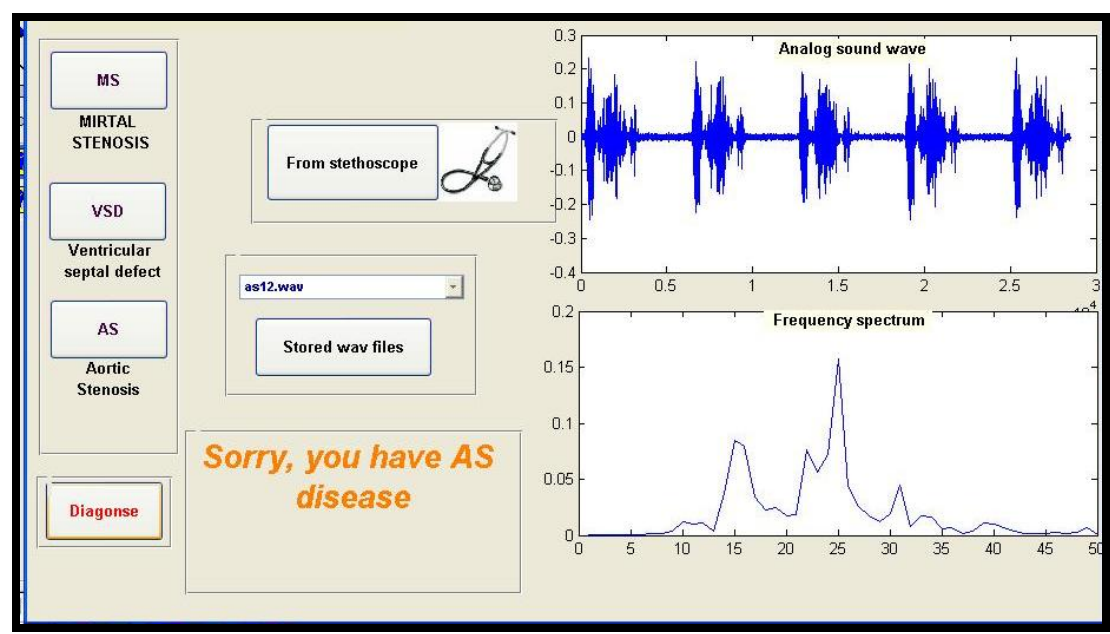

Fig 5: System Output for "aortic stenosis" heart disease.

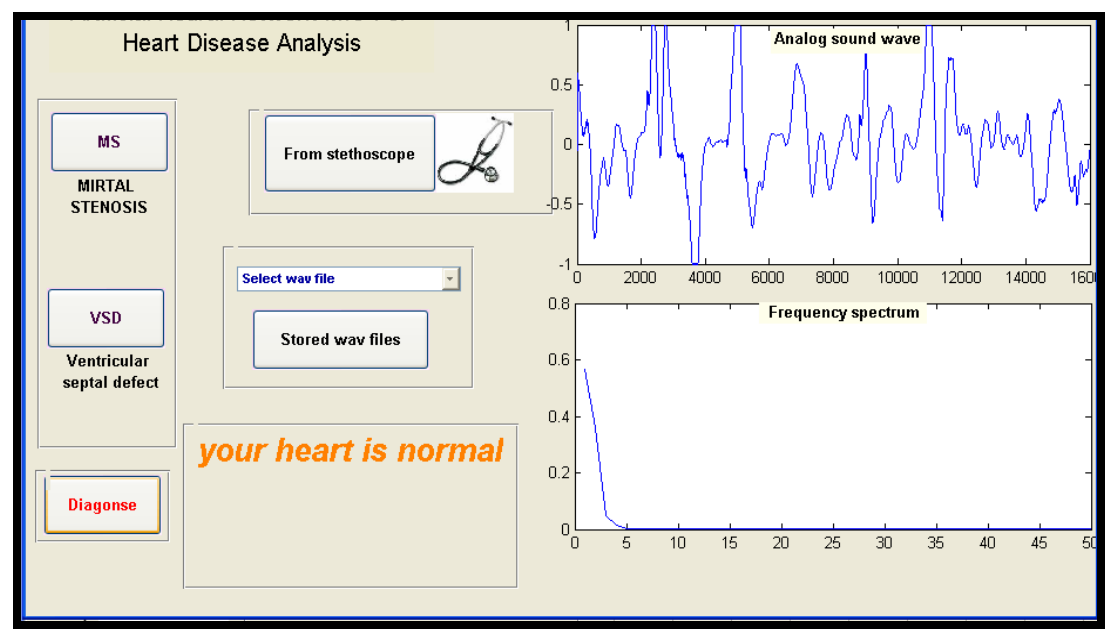

Fig 6: System Output for normal heart using stethoscope.

\section{CONCLUSION}

ANN-based innovative decision support system has been implemented and tested to classify the heart diseases: mitral stenosis, aortic stenosis and ventricular septal defect. Additionally, the system offers a promising opportunity to develop an operational screening and testing device for heart disease diagnosis and can provide great assistance for clinicians to make advanced heart sound diagnosis. Series of experiments have been conducted, using real medical data, to test the performance and accuracy of the proposed system. Compared results revealed that the system performance and accuracy are excellent, with a heart diseases classification accuracy of $92 \%$. Compared with previous studies, which are mainly focused on valvular heart diseases, results show that the proposed system delivers higher classification accuracy for both valvular and non-valvular heart diseases. Overall, the results presented that all heart diseases of MS, AS and VSD are successfully and accurately identified with the proposed system.

The proposed system can be used as great assistance to physicians in realization accurate and unfailing detecting heart problems at early stages and prevent patients going to proficient cardiologists. Further work is planned to enrich the NN knowledge base with more heart disease samples and expand the system to cover a wide range of heart diseases.

\section{ACKNOWLEDGMENTS}

The researcher gratefully acknowledges and highly appreciates the financial support and the remarkable resources provided by WISE University, Jordan.

\section{REFERENCES}

[1] Hannan, S.A., V.D. Bhagile, R.R. Manza and R.J. Ramteke, 2010. Diagnosis and medical prescription of heart disease using support vector machine and feedforward backpropagation technique. Int. J. Comput. Sci. Eng., 2: 2150-2159.

[2] Uguz, H., 2012. A biomedical system based on artificial neural network and principal component analysis for diagnosis of the heart valve diseases. J. Med. Syst., 36: 61-72.

[3] Lomsky, M., P. Gjertsson, L. Johansson, J. Richter and M. Ohlsson et al.,. 2008. Evaluation of a decision support system for interpretation of myocardial perfusion gated 
SPECT. Eur. J. Nuclear Med. Mol. Imaging, 35: 15231529.

[4] Kumar, A.V.S., 2012. Diagnosis of heart disease using fuzzy resolution mechanism. J. Artif. Intell., 5: 47-55.

[5] Al-Shayea, Q.K., 2011. Artificial neural networks in medical diagnosis. Int. J. Comput. Sci. Issues, 8: 150154

[6] Santhiyakumari, N., P. Rajendran and M. Madheswaran, 2011. Medical decision-making system of ultrasound carotid artery intima-media thickness using neural networks. J. Digital Imaging, 24: 1112-1125.

[7] Liao, Z., B. Wang, X. Xia and P.M. Hannam, 2012. Environmental emergency decision support system based on artificial neural network. Saf. Sci., 50: 150-163.

[8] Stergiou, C. and D. Siganos, 2012. Neural networks versus conventional computers. Neural Networks, http://www.doc.ic.ac.uk/ nd/surprise_96/journal/vol4/cs 11/report.html.

[9] Yana, H., Y. Jiang, J. Zheng, C. Peng and Q. Li, 2006. A multilayer perceptron-based medical decision support system for heart disease diagnosis. Expert Syst. Appl., 30: $272-281$

[10] Papik, K., B. Molnar, R. Schaefer, Z. Dombovari, Z. Tulassay and J. Feher, 2010. Application of neural networks in medicine: http://uran.donetsk.ua/ masters/2006/kita/zbykovsky/libr ary/nninmed.pdf

[11] Das, R., I. Turkoglu and A. Sengur, 2009. Diagnosis of valvular heart disease through neural networks ensembles. Comput. Methods Programs Biomed., 93: 185-191.
[12] Mehrabi, S., M. Maghsoudloo, H. Arabalibeik, R. Noormand and Y. Nozari, 2009. Application of multilayer perceptron and radial basis function neural networks in differentiating between chronic obstructive pulmonary and congestive heart failure diseases. Expert Syst. Appl., 36: 6956-6959.

[13] Tripathy, S.S., 2005. System for diagnosing valvular heart disease using heart sounds. Master's Thesis, Department of Computer Science and Engineering, Indian Institute of Technology Kanpur, India.

[14] Zamri, M.Z., S.H Salleh and M.D. Sulaiman, 2003. Wavelet analysis and classification of mitral regurgitation and normal heart sounds based on artificial neural networks. Proceedings of the 7th International Symposium on Signal Processing and Its Applications, July 1-4, 2003, Paris, France, pp: 619-620.

[15] Turkoglu, I. and A. Arslan, 2001. An intelligent pattern recognition system based on neural network and wavelet decomposition for interpretation of heart sounds. Proc. Annu. Int. Conf. IEEE Eng. Med. Biol. Soc., 2: 17471750.

[16] Turkoglu, I., A. Arslan and E. Ilkay, 2003. A wavelet neural network for the detection of heart valve diseases. Expert Syst., 20: 1-7.

[17] Kavitha, K.S., K.V. Ramakrishnan and M.K. Singh, 2010. Modeling and design of evolutionary neural network for heart disease detection. Int. J. Comput. Sci. Issues, 7: 272-283.

[18] Zin, Z.M., S. Hussain-Salleh and M.D. Sulaiman, 2003. Wavelet analysis and classification of mitral regurgitation and normal heart sounds based on artificial neural networks. Proc. Int. Symp. Signal Process. Appl., 2: 619-620. 